\title{
Quantitative Optimization of Workpiece-fixture System's Clamping Forces
}

\author{
Lu Jiping*, Zhang Faping, Zuo Jianhua \\ School of Mechanical Engineering, Beijing Institute of Technology \\ Beijing 100081, China \\ Qian Hanbo \\ The Center of Coordination \& Support of SASTIND \\ Beijing 100081, China \\ Ma Ning \\ Department of Mechanical Engineering, Karlsruhe Institute of Technology \\ Karlsruhe 76131, Germany \\ Received: 25-02-2011 \\ Accepted: 18-04-2011
}

\begin{abstract}
The paper discusses the stability of workpiece-fixture system and quantitative optimization of clamping forces during precise machining. Based on the force screw theory and the minimum norm principle, a mathematical model is formulated to calculate the entire passive forces acting on the workpiece. Furthermore, a new methodology to optimizing clamping forces is put forward, on the criteria of keeping the stability of workpiece during cutting process. By this way, the intensity of clamping forces is decreased dramatically, which is the most beneficial in improving machining quality of thin-walled machined parts. Finally, a case study is made to support and validate the proposed model.
\end{abstract}

Keywords: workpiece-fixture, system stability, clamping forces, optimization.

\section{Introduction}

Clamping force has a critical influence on the stability of workpiece-fixture system and machining precision of thin-walled part. An excessive clamping force may cause workpiece-fixture system's deflection and lower machining precision as a result, while small clamping force may result in workpiece's slippage and lower machining precision as well. Perfect clamping force should keep the force acting on the workpiece by locators positive, and lead to smaller workpiece deformation. However, clamping force is roughly estimated based on operator's experience in practice, creating random of force amplitude. Therefore, the quantitative optimization of clamping forces plays important role in the design of workpiece-fixture system $^{9,10}$.

The optimization of clamping force is one of the most important aspects in the field of machining technology. Edward from Pennsylvanian State University optimized the pre-clamping force from the point of the stiffness of workpiece-fixture system. ${ }^{1} \quad \mathrm{~B}$. $\mathrm{Li}$ optimized the clamping forces aimed at minimizing the virtual work of

* Corresponding author: lujiping661130@gmail.com 
clamping force acting on the workpiece and fixture system. $^{2}$ In order to keep the flatness of face milling process, Ying Huang optimized the side clamping force. $^{3}$

In this paper, the work force screw theory and the minimum normal force theory are used to establish the mechanical model of the workpiece-fixture system. Later clamping force is optimized aiming at minimizing normal force acting on the workpiece and constraining the stability of workpiece fixture system. Finally, a case study is made to prove the method.

\section{Mechanical model of workpiece-fixture system}

\subsection{Introduction of force screw for workpiece}

Three coordinate systems are used to analyze the force system of workpiece during cutting process, which are Global Coordinate System (GCS), Workpiece Coordinate System (WCS), and Locator Coordinate System (LCS). GCS is a fixed coordinate in three dimension space and used as a reference coordinate for others. Variable under this coordinate system is marked as G, on its up side right. WCS is fixed on the workpiece and is used to indicate the orientation of the workpiece. Variable under this coordinate system is marked as $\mathrm{W}$, on its upside right. LCS is defined at every locating point. Variable under this coordinate system is marked as L, on its upside right. Coordinates transferring among three coordinate systems can be performed by certain transfer matrix.

Any force screw is able to be decomposed in three coordinate's axis. ${ }^{4,5}$ Given a force $F^{G}$ acting on the workpeice at point $\left(P_{x}^{G}, P_{y}^{G}, P_{z}^{G}\right)$ (shown in Fig.1), the force screw caused by $F^{G}$ in GCS axis is:

$$
\mathrm{W}=\left[\begin{array}{c}
F_{x}^{G} \\
F_{y}^{G} \\
F_{z}^{G} \\
M_{x}^{G} \\
M_{y}^{G} \\
M_{z}^{G}
\end{array}\right]=\left[\begin{array}{ccc}
1 & 0 & 0 \\
0 & 1 & 0 \\
0 & 0 & 1 \\
0 & -P_{z}^{G} & P_{y}^{G} \\
P_{z}^{G} & 0 & -P_{x}^{G} \\
-P_{y}^{G} & P_{x}^{G} & 0
\end{array}\right]\left[\begin{array}{c}
F_{x}^{G} \\
F_{y}^{G} \\
F_{z}^{G}
\end{array}\right]
$$

Where $\mathrm{W}$ is the force screw. $F_{x}{ }^{G}, F_{y}{ }^{G}, F_{z}{ }^{G}$ are the decomposed forces. $M_{x}{ }^{G}, M_{y}{ }^{G}, M_{z}{ }^{G}$ are moments around three axis.

According to the force screw theory, ${ }^{6}$ the sum of all force screw acting on any balanced rigid body should be zero in three-dimension space. Namely:

$$
\sum \mathbf{w}=0
$$

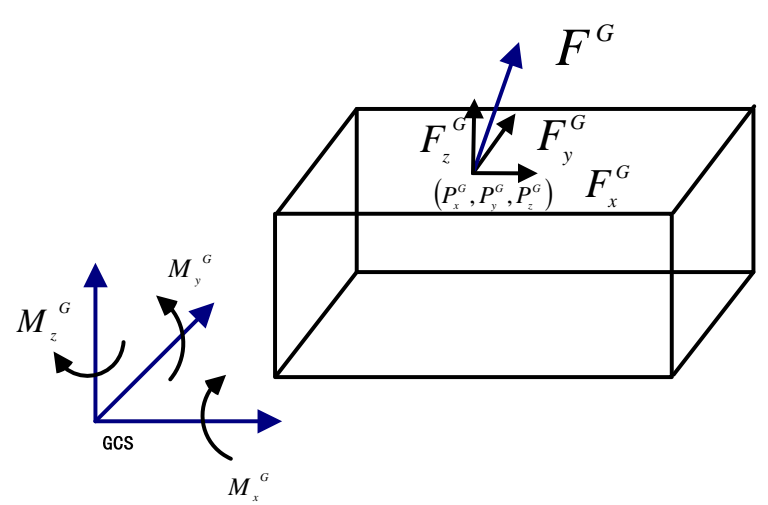

Fig. 1. Schematic of workpiece force screw

\subsection{The establishment of mechanical model for workpiece -fixture system}

\subsubsection{Contact mechanical model for workpiece}

(i) Contact mechanical model for point contact The contacts between workpiece and fixture can be dealt as point contact at larger, as shown in Fig.2. Actually, there are friction forces existing between the workpiece and locators (or clamp elements' accessorial support). ${ }^{7}$ So, the contact forces under the LCS acting on the workpiece can be written as:

$$
\mathbf{F}_{\mathbf{i}}^{\mathbf{L}}=\left[\begin{array}{lll}
F_{i x}^{L} & F_{i y}^{L} & F_{i z}^{L}
\end{array}\right]^{T} \quad(i=1,2, \ldots, n)
$$

There: $\mathrm{F}_{\mathrm{i}}^{\mathrm{L}}$ is contact force vector that the ith locator acting on the workpiece. $F_{i z}^{L}$ is the normal force of the ith locator acting on the workpiece; whereas $F_{i x}^{L}$ and $F_{i y}^{L}$ are the decomposed friction forces along the $\mathrm{x}$ and $\mathrm{y}$ axis and $n$ is the number of the locators. If the workpiece is in stability then there is no slippage between the workpiece and locators. Thus, the normal 
force and friction force should be in accord with Coulomb static friction theory:

$$
\left(F_{i x}^{L}\right)^{2}+\left(F_{i y}^{L}\right)^{2} \leq\left(\mu F_{i z}^{L}\right)^{2}
$$

Where $\mu$ is friction factor between the workpiece and

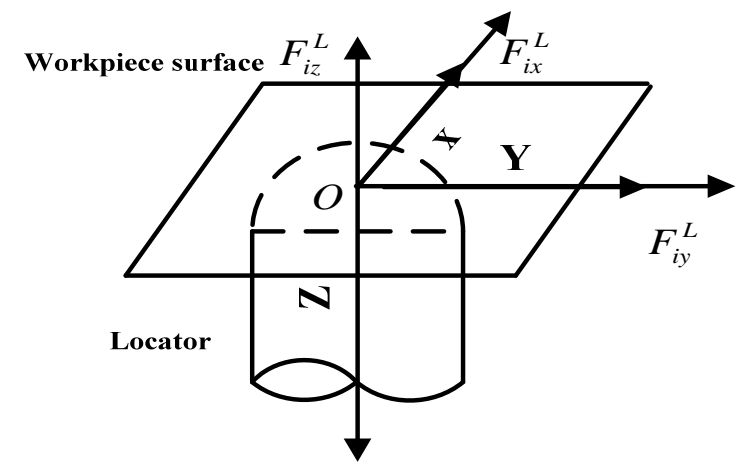

Fig. 2. Contact force model between the locator and workpiece.

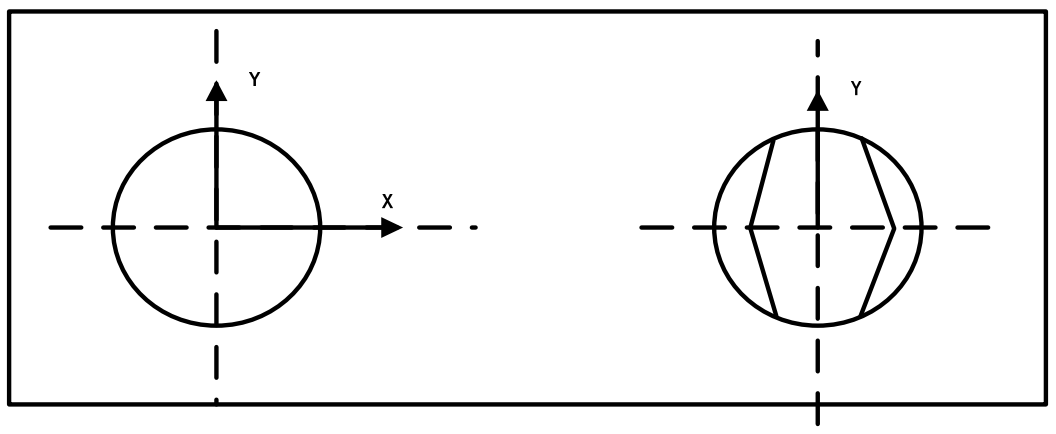

Fig. 3. Schematic of pin locator contact forces

the locator. Coulombs static friction theory is also applicable for the contact forces at clamping point on workpiece:

$$
\left(F_{j x}^{L}\right)^{2}+\left(F_{j y}^{L}\right)^{2} \leq\left(\mu F_{j z}^{L}\right)^{2} \quad(j=1,2, \cdots m)
$$

(ii) Contact mechanical model for pin locator

No friction force exists, when pin locator is used. Short pin locator is discussed here. In general short round pin and short diamond pin are used together. The following method is used to determine the force direction acting on the workpiece:

The short round pin can provide two direction forces. The first called $\mathrm{x}$ is from the intersection point between
There $F_{j z}^{L}$ is normal clamping force, $m$ is the number of clamp elements. So, by the transfer matrix they can be transferred into GCS: 
the force from short round pin under the LCS can be written as :

$$
\mathrm{F}_{\mathrm{j}}^{\mathrm{L}}=\left[\begin{array}{ll}
F_{j x}^{L} & F_{j y}^{L}
\end{array}\right]^{\mathrm{T}}(j=1,2, \ldots, m)
$$

And the force from short diamond pin under the LCS should be :

$$
\mathrm{F}_{\mathrm{j}}^{\mathrm{L}}=F_{j y}^{L} \quad(j=1,2, \ldots, m)
$$

They can be transferred to GCS by the transfer matrix respectively.

\subsubsection{Mechanical analysis for workpiece}

For general prism workpiece, there must be at least one clamping element and six locating elements to constraint its freedom and to keep the system in the stability. Given the friction is considered for $\mathrm{m}$ clamping elements and $\mathrm{n}$ locating elements, they will have $(2 m+3 n)$ passive forces acting on the workpiece at all clamping element and locating elements. As the number of passive forces will be more than 6 , the workpiece will be statically undermined. The conventional methodology-static equilibrium equations cannot be used to calculate the intensities of the reaction forces imposing on the workpiece.

According to the rigid body mechanics, the workpiece and fixture contact force problem can be resolved by employing the principle of minimum norm. ${ }^{8}$ This principle essentially states that for all possible equilibrium forces of a rigid body subjected to prescribed loading, the unique force solution compatible to the equilibrium renders a minimum force norm. As the force normal is the square of the forces, so the normal force theory is used to resolve the reaction force acting on the workpiece.

During machining process, workpiece must be in the stability by the combined action of active force (usually known) and passive force (unknown), so the forces acting on the workpiece must be a balanced force system. The active force includes cutting force, clamping force and gravitation and these forces can be called external forces. Passive forces include normal forces from locating elements, friction forces between the workpiece and clamping elements, workpiece and locating elements and these forces can be called internal force. All the internal forces make up reaction forces. So, by invoking the minimum normal principle, the following mathematical model can be achieved to calculate the reaction forces acting on the workpiece:

$$
\begin{aligned}
& \text { Minimize } \sum_{i=1}^{n}\left[\left(\mathrm{~F}_{\mathrm{i}}^{\mathrm{L}}\right)^{T}\left(\mathrm{~F}_{\mathrm{i}}^{\mathrm{L}}\right)\right]+\sum_{j=1}^{m}\left[\left(F_{j x}^{L}\right)^{2}+\left(F_{j y}^{L}\right)^{2}\right] \\
& \text { subject to } W=0 \\
& -F_{i z}^{L}<0 \quad(i=1, \cdots, n) \\
& \left(F_{i x}^{L}\right)^{2}+\left(F_{i y}^{L}\right)^{2} \leq\left(\mu F_{i z}^{L}\right)^{2} \quad(i=1, \cdots, n) \\
& \left(F_{j x}^{L}\right)^{2}+\left(F_{j y}^{L}\right)^{2} \leq\left(\mu F_{j z}^{L}\right)^{2} \quad(j=1, \cdots, m)
\end{aligned}
$$

The linear constraint of Eq. (10) describes the equilibrium state. The inequality constraints of Eq. (11) maintain that the workpiece fixture contacts are passive and unilateral, while Eqs. (12) and (13) define the tangential forces to obey Coulomb's friction law. Thus, the contact force solution is represented by a quadratic minimization with equality and inequality constraints.

A standard optimization routine may be used for the numerical solution of Eqs. (9) - (13) as a quadratic minimization with linear equality constraints and nonlinear inequality constraints, for example, the popular MATLAB system. For a typical case of practice, e.g., $n=6$ and $m=1$, it usually takes less than a few seconds to obtain a solution on a common PC.

\subsubsection{Validation of the Method}

The numerical solution using the rigid body model approach is carried out for a workpiece-fixture system. The fixture consists of a one face and two short pins localization scheme for a rectangular work-piece as shown in Fig.4, with locators L1 to L5 and clamps C1 and C2. Two hydraulic clamps are used to apply equal clamping force simultaneously.

The clamping forces at C1 and C2 are changing and a normal force $\mathrm{F}=100 \mathrm{~N}$ is imposed on the side face of the workpiece. Table 1 shows a comparison of the normal forces for each locator-workpiece contact between the predicted value and measurement value. The prediction of our model is quite agreeable with the measured data. 
Table 1 Comparison of locator normal contact forces

\begin{tabular}{ccccccccc}
\hline & \multicolumn{2}{c}{$\mathrm{C} 1=\mathrm{C} 2=500 \mathrm{~N}$} & \multicolumn{2}{c}{$\mathrm{C} 1=1000 \mathrm{~N}, \mathrm{C} 2=1500 \mathrm{~N}$} & \multicolumn{2}{c}{$\mathrm{C} 1=\mathrm{C} 2=1000 \mathrm{~N}$} & \multicolumn{2}{c}{$\mathrm{C} 1=\mathrm{C} 2=1500 \mathrm{~N}$} \\
\cline { 2 - 8 } Locator & Measured & Predicted & Measured & Predicted & Measured & Predicted & Measured & $\begin{array}{c}\text { Predicted } \\
\end{array}$ \\
\cline { 2 - 9 } & $(\mathrm{N})$ & $(\mathrm{N})$ & $(\mathrm{N})$ & $(\mathrm{N})$ & $(\mathrm{N})$ & $(\mathrm{N})$ & $(\mathrm{N})$ & $(\mathrm{N})$ \\
\hline L1 & 427.74 & 398.27 & 799.15 & 760.56 & 855.53 & 840.45 & 1283.30 & 1300.35 \\
L2 & 329.67 & 340.54 & 840.14 & 860.12 & 659.53 & 700.35 & 989.40 & 930.68 \\
L3 & 242.58 & 230.32 & 860.71 & 880.96 & 484.93 & 476.23 & 727.30 & 759.36 \\
\hline
\end{tabular}

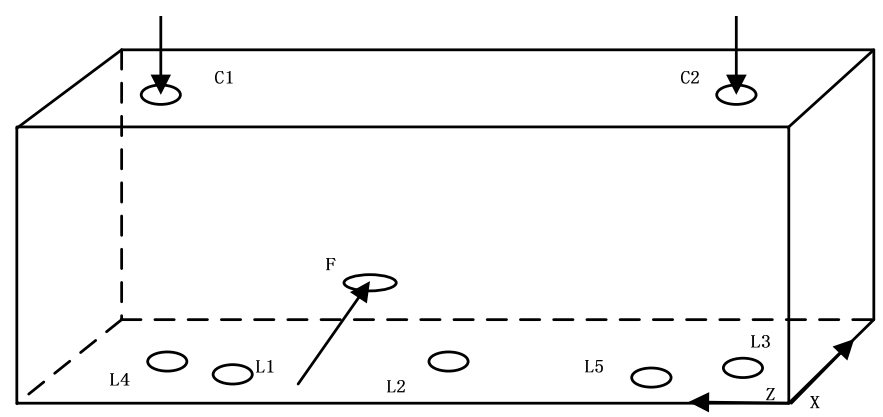

Fig. 4. The simplified workpiece-fixture system

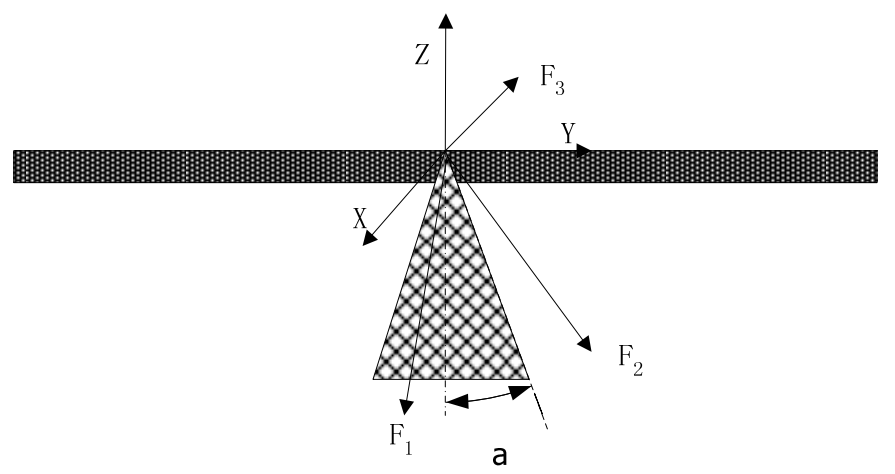

Fig. 5. Friction cone and locating stability

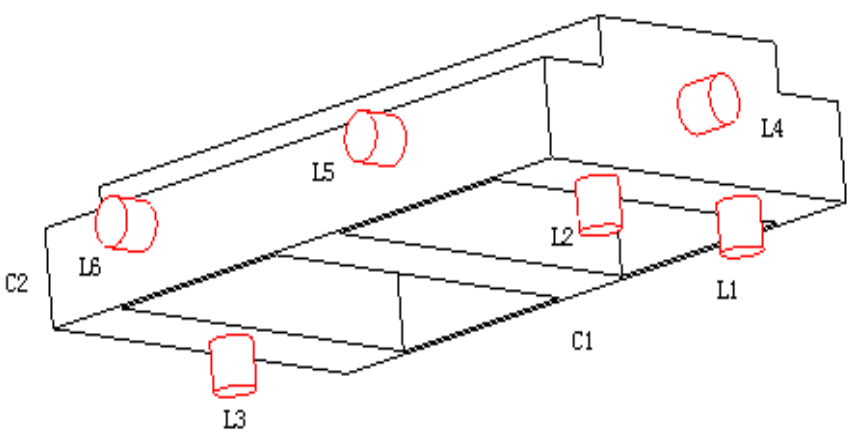

Fig. 6. The setup of workpiece-fixture system 
Table 2 cutting parameters

\begin{tabular}{llllllll}
\hline $\begin{array}{l}\text { Cut Diameter } \\
\mathrm{mm}\end{array}$ & $\begin{array}{l}\text { tooth } \\
\text { number }\end{array}$ & $\begin{array}{c}\text { feed/ } \\
(\mathrm{mm} / \text { tooth })\end{array}$ & $\begin{array}{l}\text { Spindle } \\
\text { speed/ } \\
(\mathrm{r} / \mathrm{min})\end{array}$ & $\begin{array}{l}\text { Cutting } \\
\mathrm{depth} \\
\mathrm{mm}\end{array}$ & $\begin{array}{l}\text { Cutter } \\
\text { material }\end{array}$ & $\begin{array}{l}\text { Workpiece } \\
\text { material }\end{array}$ & $\begin{array}{l}\text { Approach } \\
\text { angle/0C }\end{array}$ \\
\hline 300 & 8 & 0.15 & 458 & 1 & alloy & ZL702A & 67 \\
\hline
\end{tabular}

\section{The optimization of Clamping Forces}

If the workpiece-fixture system is under stability, all clamping forces acting on the workpiece should make any contact force between the workpiece and any locating element falls within the friction cone at the contact point (shown in Fig.5), according to the condition for a point in contact with a surface.

In the Fig. 5, 'a' is friction cone angle. There are three forces exerted onto the surface at the contact point. $F_{1}$ falls within the friction cone (shaded area), it will remain in contact with the surface. $F_{2}$ falls outside the cone but still points towards the inside of the surface, which will cause slippage; $F_{3}$ points towards outside of the surface, and it will cause the separation from the surface.

The friction cone is defined by the maximum friction force limitation:

$$
\operatorname{tg}(\alpha)=\mu
$$

Where $\mu$ is the static friction coefficient.

For convenience, the contact force is decomposed as normal force and friction force. Therefore, the normal force and friction force should obey Coulomb's friction law. During cutting process, the workpiece can be considered in stability state if any normal force and friction force obey Coulomb's friction law at any locating point. So that, considering the above force analysis for workpiece, the clamping force optimum is represented as the following:

$$
\text { Minimize } \sum_{i=1}^{n}\left[\left(\mathrm{~F}_{\mathrm{i}}^{\mathrm{L}}\right)^{T}\left(\mathrm{~F}_{\mathrm{i}}^{\mathrm{L}}\right)\right]+\sum_{j=1}^{m}\left[\left(\mathrm{~F}_{\mathrm{j}}^{\mathrm{L}}\right)^{T}\left(\mathrm{~F}_{\mathrm{j}}^{\mathrm{L}}\right)\right]
$$

Subject to

$$
\begin{gathered}
\mathbf{W}=\mathbf{0} \\
F_{i z}^{L}>0 \quad(i=1, \cdots, n) \\
F_{j z}^{L}>0 \quad(j=1, \cdots, m) \\
\left(F_{i x}^{L}\right)^{2}+\left(F_{i y}^{L}\right)^{2} \leq\left(\mu F_{i z}^{L}\right)^{2} \quad(i=1, \cdots, n) \\
\left(F_{j x}^{L}\right)^{2}+\left(F_{j y}^{L}\right)^{2} \leq\left(\mu F_{j z}^{L}\right)^{2} \quad(j=1, \cdots, m)
\end{gathered}
$$

This is a usual constrained quadratic optimization problem, so a standard optimization Matlab toolbox is used to resolve the question. It takes a few minutes to get the optimized clamping force by using Matlab in a normal PC.

\section{An illustrative example}

A thin walled workpiece and a fixture system have been employed to support the model. The setup of the workpiece-fixture system is shown in fig.6. The scheme of 3-2-1 locating has been used, in which L1 to L6 are locating points, $\mathrm{C} 1$ to $\mathrm{C} 2$ are clamping points. The initial clamping forces are: $F_{c 1}=500 N, F_{c 2}=300 N$. The top surface in fig. 6 is the machining surface with length $800 \mathrm{~mm}$ and width $300 \mathrm{~mm}$. Face milling is conducted from right side to left side of the workpiece. The cutting parameters are shown in table 2.

After the simulation of the cutting process, cutting forces acting on the workpiece at any time can be 
recorded. After that the above clamping force optimum model can be used. Finally in order to keep the workpiece fixture system in stability, the minimum needed clamping force curve of C1 and C2 clamping point can be gotten as shown in Fig.7. The abscissa is the cutting time represented as machining step. force is yielded if the equilibrium exists. Furthermore clamping force optimum model has been established, so that the oversized clamping force can be reduced and at the same time keep the workpiece-fixture system in stable state. This is very useful for the thin-walled parts. The proposed model and solution procedure are simple
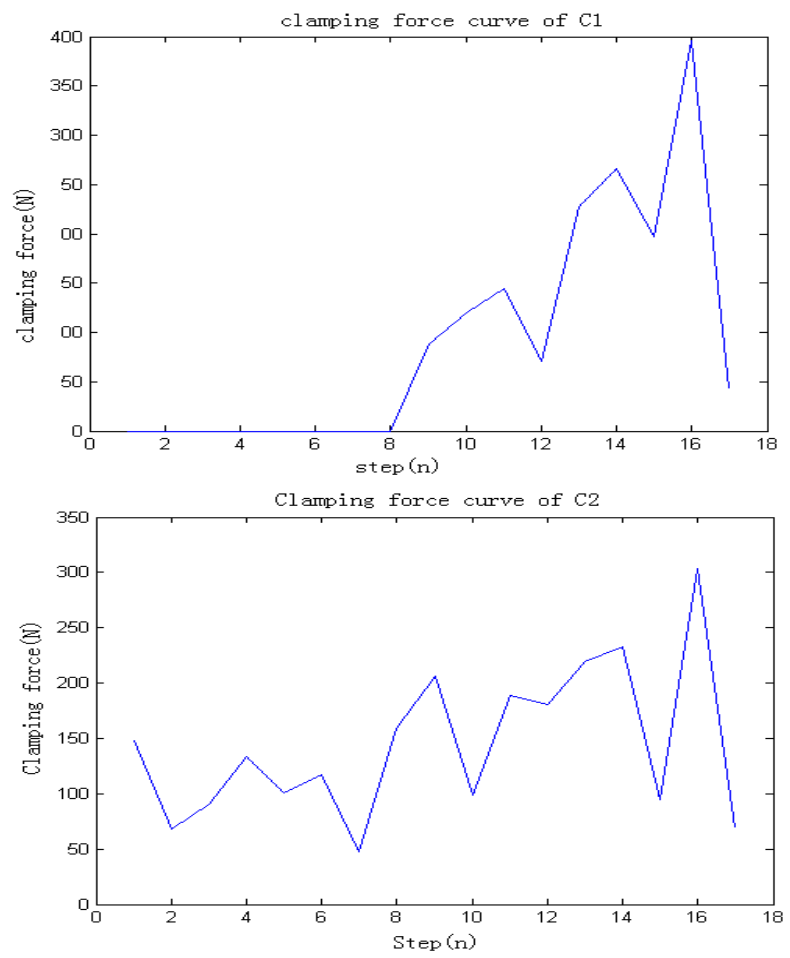

Fig. 7. Clamping force curves after optimum

From the fig. 7 we can see that, in different step the needed clamping forces at C1 and C2 points are not constant. The maximum clamping force at $\mathrm{C} 1$ point is $400 \mathrm{~N}$ (safety factor is 1.2). A decrease of $25 \%$ has been achieved compared with initial value 500N.The maximum needed clamping force at C2 point is $300 \mathrm{~N}$, and it is under $250 \mathrm{~N}$ generally.

\section{Conclusion}

The contact force model of workpiece-fixture system for different locating scheme has been established. Based on the rigid body model and the force screw theory, the contact force of workpiece-fixture has been predicted by invoking the minimum norm principle, which solves the overall model as a constraint quadratic optimization routine. The unique solution of the contact and more efficient than a model based on elastic contact properties of the workpiece-fixture system. It is particularly useful in the early stages of fixture design for fixture performance evaluation of alternative locating and clamping schemes.

\section{References}

1. De Meter Edward C. et al., A model to predict minimum required clamp pre-loads in light of fixture workpiece compliance, International Journal of Machine Tools and Manufacture, 41(7) (2001) 1031-1054.

2. B. Li, S. N. Melkote and S. Y. Liang, Analysis of Reactions and Minimum Clamping Force for Machining Fixtures with Large Contact Areas, International Journal of Advanced Manufacturing Technology, 16 (2000) 7984. 
3. Ying Huang, Tetsutaro Hoshi., Improvement of flatness error in milling plate-shaped workpiece by application of side-clamping force. Journal of the International Societies for Precision Engineering and Nanotechnology (Precision Engineering), 24 (2000) 364-370.

4. R.S.Ball, A treatise on the theory of screws (Cambridge University Press.1900).

5. K.H.Hunt, Kinematics Geometry of Mechanisms (Clarandon Press, Oxford, 1978).

6. M.S.Ohwovoriole,B.Roth., An extension of screw theory. ASME Journal of Mechanical Design, 103(1981) 725735.

7. Chou, Y-C., Chandru, V., and Barash, M. M., A Mathematical Approach to Automated Configuration of Machining Fixtures: Analysis and Synthesis, ASME J. Eng. Ind., 111(1989) 299-306.

8. Ben-Israel, A., and Greville, T. N. E., Generalized Inverses: Theory and applications (John Wiley and Sons, New York, 1974)

9. Ashfaqur Rahman, Abdus saam, Mahfusul Islam, Partha Sarker, An Image Based Approach to Compute Object Distance, International Journal of Computational Intelligence Systems, Vol.1, No.4, (December 2008), 304-312

10. Hyung Joon Kook, Geometry Tutoring Supported by an Intelligent Drawing Interface and Automatic Problem Solving, International Journal of Computational Intelligence Systems, Vol.3, No.1, (April 2010), 21-27 